\title{
Testing mediation effects in cross-classified multilevel data
}

\author{
Wen Luo ${ }^{1}$
}

Published online: 16 March 2016

(C) Psychonomic Society, Inc. 2016

\begin{abstract}
In this article, we propose an approach to test mediation effects in cross-classified multilevel data in which the initial cause is associated with one crossed factor, the mediator is associated with the other crossed factor, and the outcome is associated with Level-1 units (i.e., the $2^{(\mathrm{A})} \rightarrow 2^{(\mathrm{B})} \rightarrow 1$ design). Multiple-membership models and cross-classified random effects models are used to estimate the indirect effects. The method is illustrated using real data from the Early Childhood Longitudinal Study-Kindergarten Cohort (1998). The results from the simulation study show that the proposed method can produce a consistent estimate of the indirect effect and reliable statistical inferences, given an adequate sample size.
\end{abstract}

Keywords Mediation analysis - Cross-classified multilevel data $\cdot$ Multiple membership models $\cdot$ Cross-classified random effects models

Testing mediation effects has an important role in social and behavioral research because researchers are often interested in knowing the pathways through which the effect of the initial cause is transferred to the outcome. In recent years, with the development of multilevel modeling techniques such as multilevel linear regression and multilevel structural equation modeling (MSEM), mediation analysis has been extended from single-level to multilevel data.

Wen Luo

wluo@email.tamu.edu

1 Educational Psychology, Texas A\&M University, College Station, TX, USA
In two-level nested data, Krull and MacKinnon (2001) first examined three types of multilevel mediation designs using hierarchical linear models (HLMs): the $1 \rightarrow 1 \rightarrow 1$ design, in which the initial predictor, the mediator, and the outcome are all measured at Level 1 (or the lowest level); the $2 \rightarrow 1 \rightarrow 1$ design, in which the initial predictor is at Level 2 (or the cluster level), and the mediator and outcome are at Level 1; and the $2 \rightarrow 2 \rightarrow 1$ design, in which the initial predictor and the mediator are at Level 2 and the outcome is at Level 1. Because the effect of a Level-1 predictor could be randomly varying across Level-2 clusters, researchers have further developed procedures for testing random indirect effects in the $1 \rightarrow 1 \rightarrow 1$ design (e.g., Bauer, Preacher, \& Gil, 2006; Kenny, Korchmaros, \& Bolger, 2003).

Zhang, Zyphur, and Preacher (2009) pointed out that Krull and MacKinnon's (2001) simple reformulations of singlelevel techniques may produce biased estimates of the multilevel mediation effects, due to the conflated between-cluster and within-cluster effects in typical HLM models. Zhang et al. recommended the use of the group-mean centering approach (or centering within a context) to decompose some multilevel mediation effects into Level-1 and Level-2 effects for clearer interpretation. However, it has been shown that the estimate of the between-level effect may be biased when using the cluster mean as the Level-2 predictor, due to insufficient reliability in the observed cluster means (Asparouhov \& Muthen, 2006).

More recently, Preacher, Zyphur, and Zhang (2010) integrated the existing methods into a unified MSEM framework. Unlike the HLM framework, the MSEM framework has two advantages for assessing multilevel mediation effects. First, Level-2 outcomes are easily accommodated. Mediation models that cannot be analyzed in the multilevel linear regression framework, such as $1 \rightarrow 1 \rightarrow 2$ and $1 \rightarrow 2 \rightarrow 2$, can be accommodated in the MSEM framework. Second, the between- and within-level components of indirect effects can be estimated 
separately without using the group-mean centering approach. MSEM uses the latent-variable approach to provide a less biased estimate of the between-level effects.

So far, the extant literature on multilevel mediation analysis has dealt exclusively with data with strictly nested structures. In reality, however, multilevel data may not always be strictly nested. For example, students may be cross-classified by schools and neighborhoods, because students attending the same school may live in different neighborhoods, and students living in the same neighborhood may attend different schools. A variety of mediation designs can be used with crossclassified multilevel data. We first review three common designs that are direct extensions of their counterparts in strictly nested data. First, the $1 \rightarrow 1 \rightarrow 1$ design can be extended to the situation in which Level-1 units are cross-classified by two Level-2 random factors. For example, the indirect effect of students' cognitive ability on their academic achievement through parental expectations may be investigated using data in which the students are cross-classified by schools and neighborhoods. Second, the $2 \rightarrow 1 \rightarrow 1$ design can be extended to the $2^{(\mathrm{A})} \rightarrow 1 \rightarrow 1$ design, where $2^{(\mathrm{A})}$ represents one of the crossed factors at Level 2. For example, controlling for school random effects, neighborhood social distress may affect students' hopefulness, which subsequently affects their involvement in violence. Third, the $2 \rightarrow 2 \rightarrow 1$ design can be extended to the $2^{(\mathrm{A})} \rightarrow 2^{(\mathrm{A})} \rightarrow 1$ design, in which the initial predictor and the mediator are both associated with one of the crossed factors. For example, controlling for neighborhood random effects, school ethnic and academic composition may have indirect effects on students' achievement via the school's educational climate (Brault, Janosz, \& Archambault, 2014).

In addition to the three common designs directly borrowed from nested data, a fourth design is unique to cross-classified data - that is, the $2^{(\mathrm{A})} \rightarrow 2^{(\mathrm{B})} \rightarrow 1$ design. In this design, the initial cause is associated with one of the crossed factors, and the mediator is associated with the other. An example of such a design is that neighborhood characteristics such as high-status residents and residential stability can influence school characteristics such as the school atmosphere, which subsequently affect students' achievement (Ainsworth, 2002). Another example of such a design can be found in the medical setting, where doctors' respect for nurses affects the nurses' job satisfaction, which subsequently affects patients' wellbeing. Patients are often cross-classified by doctors and nurses, because patients under the same doctor may be cared for by different nurses and patients cared by the same nurse may belong to different doctors. In this case, doctors and nurses are two crossed random factors.

To test indirect effects in the first three designs, researchers can use the cross-classified structural equation models (CCSEMs) in the MSEM framework (Asparouhov \& Muthén, 2012). However, the analysis is more challenging for the fourth design $\left(2^{(\mathrm{A})} \rightarrow 2^{(\mathrm{B})} \rightarrow 1\right)$, especially for the estimation of the
$2^{(\mathrm{A})} \rightarrow 2^{(\mathrm{B})}$ path, which represents the effect of one crossed factor on the other. This is because when factor A and factor $\mathrm{B}$ are crossed, a unit in factor B may be influenced by multiple units in factor A simultaneously. For example, when schools and neighborhoods are crossed, a school may be affected by multiple neighborhoods simultaneously. In this case, a school is a member of multiple neighborhoods. When modeling the effect of neighborhoods on schools, in this case, it is necessary to estimate the overall effect of multiple neighborhoods on a school while taking into consideration the variation in neighborhood random effects. Such a design cannot be handled by cross-classified SEMs, because the variables associated with one crossed factor cannot be used in the structural equations specified for the other crossed factor.

To solve this problem, we propose a method that uses the multiple-membership (MM) model and the cross-classified random effects $(\mathrm{CC})$ model to estimate and test the indirect effects in the $2^{(\mathrm{A})} \rightarrow 2^{(\mathrm{B})} \rightarrow 1$ design. It should be noted that in a rare situation, in observational studies where the data are completely balanced (i.e., equal numbers of Level-1 units in each cell), the $2^{(\mathrm{A})} \rightarrow 2^{(\mathrm{B})}$ path cannot be assessed because in this case the two factors are orthogonal. Therefore, the proposed method is only applicable for unbalanced crossclassified multilevel data.

In the following sections, we briefly show how the first three common designs are analyzed in the MSEM framework. Then we describe the proposed method to estimate indirect effects in the fourth design (i.e., $2^{(\mathrm{A})} \rightarrow 2^{(\mathrm{B})} \rightarrow 1$ ) and demonstrate the method using data from the Early Childhood Longitudinal Study. In addition, we conducted a simulation study to assess the performance of the method in terms of the biases in the parameter estimates and the statistical inferences.

\section{Estimating indirect effects in the $1 \rightarrow 1 \rightarrow 1,2^{(A)} \rightarrow 1 \rightarrow 1$, and $2^{(\mathrm{A})} \rightarrow 2^{(\mathrm{A})} \rightarrow 1$ designs}

To test indirect effects in the first three designs, researchers can use cross-classified SEMs in the MSEM framework. In a cross-classified SEM, the Level-1 observed variables are implicitly decomposed into latent components at the individual level (e.g., students), the classification A level (e.g., neighborhoods), and the classification B level (e.g., schools). Separate structural equations are defined for the three levels and estimated simultaneously. The cross-classified SEM can be fitted with Mplus version 7 (Muthén \& Muthén, 1998-2012) using the Bayesian estimation method. Exemplary Mplus syntax is provided in Appendix A for each of the three designs.

In the $1 \rightarrow 1 \rightarrow 1$ design, the indirect effect can be decomposed into the within-level, school-level, and neighborhood-level components. In the current version of Mplus (i.e., Mplus 7.0), a Level-1 predictor cannot be automatically decomposed into latent components in cross- 
classified SEMs, because Mplus does not allow us to use a Level-1 predictor in equations at higher crossed levels. Therefore, to obtain the latent component of a Level-1 predictor at a specific level, we have to specify a latent variable at the corresponding level, using the observed predictor as the sole indicator whose factor loading is constrained to 1 and residual variance is constrained to 0 . For example, in the syntax for the $1 \rightarrow 1 \rightarrow 1$ design, predictor $x$ is decomposed to the within-level (fx_w), school-level (fx_s), and neighborhood-level (fx_n) latent components. The same decomposition is also applied to the mediator $m$. Then, in the within-level model, the latent component of $m$ (fm_w) is regressed on the latent component of $x$ (fx_w) to obtain the within-level estimate of the $a$ path (aw). The outcome $y$ is then regressed on the within-level latent components of $x$ (fx_w) and $m$ (fm_w) to obtain the within-level estimate of the $b$ path (bw). Finally, the withinlevel indirect effect is obtained by multiplying aw and bw. In the same vein, the school-level and neighborhood-level indirect effects are specified in the models.

On the other hand, in the $2^{(\mathrm{A})} \rightarrow 1 \rightarrow 1$ design, because the initial predictor is associated with neighborhood, the indirect effect is only defined at the neighborhood level. Therefore, we only need to obtain the neighborhood-level latent component of the mediator $m$ and specify the indirect effect at the neighborhood level. Similarly, in the $2^{(\mathrm{A})} \rightarrow 2^{(\mathrm{A})} \rightarrow 1$ design, both the initial predictor and the mediator are at the neighborhood level; therefore, the indirect effect exists only at the neighborhood level, and no decomposition of the initial predictor or the mediator is needed.

Note that CC-SEM can also accommodate designs in which the initial predictor is at the individual level, whereas the mediator and/or the outcome are associated with one of the upper levels (e.g., $1 \rightarrow 2^{(\mathrm{A})} \rightarrow 2^{(\mathrm{A})}, 1 \rightarrow 1 \rightarrow 2^{(\mathrm{A})}, 1 \rightarrow 2^{(\mathrm{A})} \rightarrow 1$ ). To save space, we will not elaborate on these designs, because such designs are less common in real applications, and the same approach to latent component decomposition can be used in Mplus.

\section{Estimating the indirect effect in the $2^{(\mathrm{A})} \rightarrow 2^{(\mathrm{B})} \rightarrow 1$ design}

Although CC-SEM is flexible and offers many modeling possibilities, it cannot handle the $2^{(\mathrm{A})} \rightarrow 2^{(\mathrm{B})} \rightarrow 1$ design. Therefore, we propose a method to estimate indirect effects in such a design. The proposed method consists of three steps. First, an MM model is used to estimate the $2^{(\mathrm{A})} \rightarrow 2^{(\mathrm{B})}$ path. Second, a CC model is used to estimate the $2^{(\mathrm{B})} \rightarrow 1$ path. Finally, the indirect effect is computed as the product of the two path coefficients and its confidence interval is obtained. Below, we describe how the MM and $\mathrm{CC}$ models are specified to estimate the $2^{(\mathrm{A})} \rightarrow 2^{(\mathrm{B})}$ and the $2^{(\mathrm{B})} \rightarrow 1$ paths, respectively.

\section{MM model to estimate the $2^{(\mathrm{A})} \rightarrow 2^{(\mathrm{B})}$ path}

As an extension of standard HLMs, MM models deal with the case in which a lower-level unit is a member of more than one higher-level unit (Browne \& Goldstein, 2001). For example, individuals may change residency over time, so they are often members of multiple neighborhoods (e.g., Chandola, Clarke, Wiggins, \& Bartley, 2005). In cross-classified data, the two random factors $\mathrm{A}$ and $\mathrm{B}$ also have an MM relationship. For example, when schools and neighborhoods are crossed, a school draws students from multiple neighborhoods; therefore, the school can be viewed as a member of multiple neighborhoods, and each neighborhood has an impact on the school.

An MM model allows us to estimate the overall effect of neighborhoods on schools (i.e., the $2^{(\mathrm{A})} \rightarrow 2^{(\mathrm{B})}$ path) while taking into consideration the random neighborhood effects. In addition, MM models allow us to take the relative importance of the neighborhoods into consideration. For example, neighborhoods sending more students to the school may have a greater impact than neighborhoods sending fewer students to the school. To incorporate this information into the model, a weighting scheme is used, so that the total impact of the multiple neighborhoods is a weighted sum of the individual neighborhoods' effects. One way of choosing the weights is to use the proportion of a school's students living in each neighborhood. Below we present the detailed formulation of the MM model used to estimate the $2^{(\mathrm{A})} \rightarrow 2^{(\mathrm{B})}$ path.

Let $M$ be the mediator associated with schools and $X$ be the initial predictor associated with neighborhoods. The model is specified as follows:

$$
\begin{aligned}
M_{j}= & \beta_{0}+\beta_{1} \sum_{k \in \operatorname{Neighborhood}(j)} w_{j k} X_{k} \\
& +\sum_{k \in \operatorname{Neighborhood}(j)} w_{j k} \mu_{k}+r_{j},
\end{aligned}
$$

where $j$ is the index of schools, $k$ is the index of neighborhoods, Neighborhood $(j)$ represents the set of neighborhoods from which school $j$ draws students, $w_{j k}$ represents the weight of neighborhood $k$ associated with school $j, \mu_{k}$ represents the random effect of neighborhood $k\left[\mu_{k} \sim \mathrm{N}\left(0, \sigma_{\mu}^{2}\right)\right]$, and $r_{j}$ represents the error associated with school $j\left[r_{j} \sim \mathrm{N}\left(0, \sigma_{r}^{2}\right)\right]$. The regression coefficient $\beta_{1}$ is the effect of $X$ on $M$ - that is, the coefficient of the $2^{(\mathrm{A})} \rightarrow 2^{(\mathrm{B})}$ path.

\section{CC models to estimate the $2^{(\mathrm{B})} \rightarrow 1$ path}

To estimate the direct effect of the mediator on the outcome, controlling for the initial predictor (i.e., the $2^{(\mathrm{B})} \rightarrow 1$ path), we can use a CC model (Browne \& Goldstein, 2001; Rasbash \& Goldstein, 1994). As an extension to the standard HLMs, CC 
models allow the random effects of multiple crossed factors (e.g., schools and neighborhoods) to be estimated simultaneously.

Let $Y$ be the outcome variable at the student level. The CC model is specified as follows:

$Y_{i(j k)}=\beta_{0}+\beta_{2} M_{j}+\beta_{3} X_{k}+\delta_{j}+\tau_{k}+e_{i(j k)}$,

where $i$ is the index of students, $j$ is the index of schools, $k$ is the index of neighborhoods. $\delta_{j}$ represents the random effect of schools $\left[\delta_{j} \sim \mathrm{N}\left(0, \sigma_{\delta}^{2}\right)\right], \tau_{k}$ represents the random effect of neighborhood $\left[\tau_{k} \sim \mathrm{N}\left(0, \sigma_{\tau}^{2}\right)\right]$, and $e_{i}(j k)$ represents the error associated with students $\left[e_{i}(j k) \sim \mathrm{N}\left(0, \sigma_{e}^{2}\right)\right]$. The regression coefficient $\beta_{2}$ represents the direct effect of $M$ on $Y$ controlling for $X$ - that is, the coefficient for the $2^{(\mathrm{B})} \rightarrow 1$ path.

Both the MM and CC models can be estimated using the maximum likelihood estimation method. Many computer programs, such as SAS PROC MIXED, Stata xtmixed, and R lme4, can fit these models.

\section{Confidence intervals of the indirect effect}

On the basis of the estimates from Eqs. 1 and 2, the indirect effect is computed as the product of $\beta_{1}$ and $\beta_{2}$ (i.e., $\beta_{1} \beta_{2}$ ). The confidence intervals of the indirect effect can be computed with the standard methods. In this study, we focus on the analytical approach and the Monte Carlo approach. Using the analytical approach, the standard error of the indirect effect can be computed using the following formula, given multivariate normality (Baron \& Kenny, 1986):

$S_{\beta_{1} \beta_{2}}=\sqrt{\beta_{1}^{2} S_{\beta_{2}}^{2}+\beta_{2}^{2} S_{\beta_{1}}^{2}+S_{\beta_{1}}^{2} S_{\beta_{2}}^{2}}$,

where $S_{\beta_{1}}^{2}$ and $S_{\beta_{2}}^{2}$ are the variance of $\beta_{1}$ and $\beta_{2}$, respectively. The confidence interval of the indirect effect is then computed as

$C I_{1-\alpha}=\beta_{1} \beta_{2} \pm z_{\alpha / 2} S_{\beta_{1} \beta_{2}}$.

In addition to the analytical approach, there is also the Monte Carlo approach for computing the confidence intervals of indirect effects (MacKinnon, Lockwood, \& Williams, 2004). This method assumes that $\beta_{1}$ and $\beta_{2}$ have a bivariate normal distribution. The bivariate normal distribution is simulated using the parameter estimates as the means and their asymptotic variances and covariance (i.e., $\left[\begin{array}{l}\beta_{1} \\ \beta_{2}\end{array}\right] \sim \mathrm{N}\left[\begin{array}{l}\hat{\beta}_{1} \\ \hat{\beta}_{2}\end{array}\right]$ $\left[\begin{array}{ll}\hat{S}_{\beta_{1}}^{2} & \\ \hat{S}_{\beta_{1}, \beta_{2}} & \hat{S}_{\beta_{2}}^{2}\end{array}\right]$ ). Note that in the $2^{(\mathrm{A})} \rightarrow 2^{(\mathrm{B})} \rightarrow 1$ design, the asymptotic covariance between $\beta_{1}$ and $\beta_{2}\left(S_{\beta_{1}, \beta_{2}}\right)$ is 0 , because the mediator and the outcome are at different levels and their residuals (i.e., $r_{j}$ and $e_{i}(j k)$ ) are uncorrelated. Thousands of random draws of $\beta_{1}$ and $\beta_{2}$ are simulated, and the product $\beta_{1} \beta_{2}$ is computed. The resulting distribution of $\beta_{1}$ $\beta_{2}$ is then used to estimate the lower and upper boundaries of the confidence interval for $\beta_{1} \beta_{2}$. Computer software is available to compute the Monte Carlo confidence interval (e.g., Selig \& Preacher, 2008).

\section{Sequential versus simultaneous estimation}

The proposed method sequentially estimates the $2^{(\mathrm{A})} \rightarrow 2^{(\mathrm{B})}$ and the $2^{(\mathrm{B})} \rightarrow 1$ paths in two separate models, rather than simultaneously estimating them in one model. It is possible to use the simultaneous estimation approach (e.g., Bauer et al., 2006), which employs selection (or dummy) variables to combine the two separate models into a single equation. The simultaneous estimation approach allows us to obtain the asymptotic covariance between $\beta_{1}$ and $\beta_{2}$, which is important for the computation of the confidence intervals of the indirect effect. In the $2^{(\mathrm{A})} \rightarrow 2^{(\mathrm{B})} \rightarrow 1$ design, however, the asymptotic covariance between $\beta_{1}$ and $\beta_{2}$ is always 0 ; therefore, there is no additional benefit of using the simultaneous estimation approach. To verify this, we analyzed empirical data and simulated data using both the sequential and the simultaneous estimation approaches, finding that the results were identical.

\section{Demonstration}

To demonstrate the proposed method, we used data from the Early Childhood Longitudinal Study-Kindergarten Cohort (1998). Student, school, and neighborhood data from the spring of 1999 were extracted. The indirect effect of neighborhood high-status residents on student achievement via school academic atmosphere was examined. The analysis was conducted using SAS 9.4, and the syntax for the data preparation and model fitting is included in Appendix B.

\section{Description of the sample of analysis}

The sample data have a cross-classified multilevel structure in which students are jointly nested within schools and neighborhoods (defined by zip code tabulation areas, or ZCTAs). In addition, schools and neighborhoods are crossed. After deleting missing data and schools with fewer than 20 students, to avoid nonconvergence in the estimation, the final sample of analysis consisted of 2,771 students, cross-classified by 130 schools and 128 neighborhoods. 


\section{Measures}

The outcome variable was students' math scores, computed on the basis of item response theory in the spring of 1999 . The initial predictor was neighborhood high-status residents (NHSR), and the mediator was school academic atmosphere. Below we describe how the NHSR and school atmosphere variables were created.

Neighborhood high-status residents Following Ainsworth (2002), we created standardized composite scores based on (1) proportions of college graduates among persons over 25 years of age and (2) proportions of employed persons with professional or managerial occupations among people over 16 years of age. This variable was used as the predictor $(X)$ in the mediation model.

School academic atmosphere In the administrator questionnaire of the ECLS-K study, 11 items measure how much emphasis the administrator places on certain goals and objectives for kindergarten teachers, such as facilitating children's progress in language and number skills, maintaining a quiet and orderly class environment, and so forth. We computed the total scores and used them as mediators $(M)$ in the model. Because the distribution of school climate scores was skewed, we applied the square root transformation to make the distribution better approximate normality.

\section{Analysis}

To estimate the $2^{(\mathrm{A})} \rightarrow 2^{\text {(B) }}$ path, we first restructure the data and compute the weight of each neighborhood associated with each school. To illustrate the process, we use a small hypothetical dataset with 11 students cross-classified by two schools and three neighborhoods. In Table 1, columns 1, 2, and 3 are the student, school, and neighborhood IDs, respectively. The next three columns are the variables associated with neighborhoods $(X)$, schools $(M)$, and students $(Y)$, respectively. The data are first aggregated to obtain the frequency of students in each school-neighborhood combination (or cell). The aggregated data are presented in Table 2. On the basis of the frequency in column 3 , the weight of each neighborhood associated with each school is computed (see column 4). For example, the weight of Neighborhood 1 associated with School 1 in the first row is 0 , because no student in School 1 comes from Neighborhood 1. The weight of Neighborhood 2 associated with School 1 is $2 / 5=.4$, because two out of the five students in School 1 come from Neighborhood 2. The weight of Neighborhood 3 associated with School 1 is $3 / 5=.6$, because three out of the five students in School 1 come from Neighborhood 3. These three weights add up to 1 .
Table 1 A small, hypothetical cross-classified dataset

\begin{tabular}{llllll}
\hline Stu_ID & Sch_ID & Nei_ID & $X$ & $M$ & $Y$ \\
\hline 1 & 1 & 2 & 8 & 4 & 5 \\
2 & 1 & 2 & 8 & 4 & 7 \\
3 & 1 & 3 & 9 & 4 & 4 \\
4 & 1 & 3 & 9 & 4 & 2 \\
5 & 1 & 3 & 9 & 4 & 8 \\
6 & 2 & 1 & 7 & 5 & 9 \\
7 & 2 & 1 & 7 & 5 & 1 \\
8 & 2 & 1 & 7 & 5 & 3 \\
9 & 2 & 1 & 7 & 5 & 4 \\
10 & 2 & 2 & 8 & 5 & 7 \\
11 & 2 & 2 & 8 & 5 & 10 \\
\hline
\end{tabular}

Multiplying Weight and $X$ (i.e., $w_{j k} X_{k}$ ) and adding up across the set of neighborhoods associated with a school (i.e., $\sum_{k \in \text { Neighborhood }}(j) w_{j k} X_{k}$ ), we obtain the weighted $X$. For example, the weighted $X$ corresponding to School 1 is $8.6(0 * 7+.4 * 8+.6 * 9)$. This weighted $X$ represents the composite characteristic of the set of neighborhoods that have impacts on School 1.

Finally, the data are restructured to wide form, as is shown in Table 3. The weights of the three neighborhoods become three weight variables (W1, W2, and W3). Using the restructured data, the MM model is fitted with $M$ as the outcome variable and the weighted $X$ as the predictor. The weight variables have random effects with a common variance.

Next, using the original data shown in Table 1 , the $2^{(\mathrm{B})} \rightarrow 1$ path is estimated using the $\mathrm{CC}$ model with $Y$ as the outcome and $X$ and $M$ as the predictors. Finally, the two path coefficients and their standard errors are used to compute the indirect effect and the analytical and Monte Carlo confidence intervals.

\section{Results}

Table 4 shows the parameter estimates of the MM model. Because all of the variables were standardized, the estimates can be viewed as standardized coefficients. The estimated $2^{(\mathrm{A})} \rightarrow 2^{(\mathrm{B})}$ path $\left(\beta_{1}\right)$ is .450 with a standard error of .089 .

Table 2 Hypothetical school and neighborhood data with weights

\begin{tabular}{lllllll}
\hline Sch_ID & Nei_ID & Frequency & Weight & $X$ & $M$ & Weighted $X$ \\
\hline 1 & 1 & 0 & 0 & 7 & 4 & 8.6 \\
1 & 2 & 2 & .4 & 8 & 4 & 8.6 \\
1 & 3 & 3 & .6 & 9 & 4 & 8.6 \\
2 & 1 & 4 & .67 & 7 & 5 & 7.33 \\
2 & 2 & 2 & .33 & 8 & 5 & 7.33 \\
2 & 3 & 0 & 0 & 9 & 5 & 7.33 \\
\hline
\end{tabular}


Table 3 Restructured school and neighborhood data with weights

\begin{tabular}{llllll}
\hline Sch_ID & W1 & W2 & W3 & Weighted $X$ & $M$ \\
\hline 1 & 0 & .4 & .6 & 8.6 & 4 \\
2 & .67 & .33 & 0 & 7.33 & 5
\end{tabular}

Table 5 shows the standardized parameter estimates of the CC model. The estimated $2^{(\mathrm{B})} \rightarrow 1$ path $\left(\beta_{2}\right)$ is .079 with a standard error of .038. Multiplying $\beta_{1}$ by $\beta_{2}$, we obtain the standardized indirect effect estimate as .036 . On the basis of Eqs. 3 and 4 , the analytical $95 \%$ confidence interval is [-.001, .072], indicating a nonsignificant indirect effect. On the other hand, the Monte Carlo $95 \%$ confidence interval is [.002, .076], indicating a significant indirect effect. It is not surprising that the two approaches yield different results, because the analytical approach assumes a normal sampling distribution of the indirect effects, whereas the sampling distribution obtained using the Monte Carlo approach was slightly positively skewed.

\section{Simulation study}

We conducted a simulation study to examine whether the proposed method can produce consistent estimates of indirect effects and reliable confidence intervals under various data conditions. We chose three major design factors: sample size, degree of cross-classification, and effect size.

\section{Design factors}

Sample size The extant literature has demonstrated that sample size has a significant impact on multilevel model estimation (e.g., Maas \& Hox, 2005). To examine the performance of the proposed method under small, moderate, and large sample sizes, we selected three conditions: (1) 200 students crossclassified by 20 schools and 20 neighborhoods, (2) 500 students cross-classified by 50 schools and 50 neighborhoods, and (3) 2,000 students cross-classified by 100 schools and 100 neighborhoods.

Table 4 Multiple-membership model estimates

\begin{tabular}{llllll}
\hline Parameters & Estimates & $S E$ & $d f$ & $t$ & $p$ \\
\hline Fixed Effects & & & & & \\
$\quad$ Weighted $X\left(\beta_{1}\right)$ & .45 & .089 & 121 & 5.07 & $<.001$ \\
Variance Components & & & & & \\
$\quad$ Neighborhoods $\left(\sigma_{\mu}^{2}\right)$ & .174 & .131 & & & \\
$\quad$ Residual $\left(\sigma_{r}^{2}\right)$ & .670 & .136 & & & \\
\hline
\end{tabular}

Table 5 Cross-classified random effects model estimates

\begin{tabular}{llllll}
\hline Parameters & Estimates & $S E$ & $d f$ & $t$ & $p$ \\
\hline Fixed Effects & & & & & \\
$\quad M\left(\beta_{2}\right)$ & .079 & .038 & 126 & 2.07 & .040 \\
$X\left(\beta_{3}\right)$ & .207 & .038 & 114 & 5.39 & $<.001$ \\
Variance Components & & & & & \\
$\quad$ Schools $\left(\sigma_{\delta}^{2}\right)$ & .105 & .021 & & & \\
$\quad$ Neighborhoods $\left(\sigma_{\tau}^{2}\right)$ & .014 & .025 & & & \\
$\quad$ Residual $\left(\sigma_{e}^{2}\right)$ & .819 & .023 & & & \\
\hline
\end{tabular}

Degree of cross-classification Researchers have shown that the degree of cross-classification may have an impact on the parameter estimates in CC models (Luo, Cappaert, \& Ning, 2015). In addition, because the $2^{(\mathrm{A})} \rightarrow 2^{(\mathrm{B})}$ path cannot be assessed when the data are completely balanced, it was important to evaluate the performance of the method when the degree of crossclassification was high. Hence, we considered two conditions: (1) the fully cross-classified condition (or high degree of cross-classification), and (2) the partially crossclassified condition (or low degree of cross-classification). In the fully cross-classified condition, the students in a neighborhood had equal probabilities to be assigned to any of the schools, and vice versa. In the partially cross-classified condition, $20 \%$ of the neighborhoods were selected, and the students in each selected neighborhood were randomly assigned to only two schools. For the remaining neighborhoods, there was a one-toone match between schools and neighborhoods.

Effect size We selected two levels for the effect size of the indirect effect. The small effect was based on the parameter estimates from the empirical data analysis $\left(\beta_{1}=.5\right.$ and $\beta_{2}=$ $.1)$. For the large effect, we set $\beta_{1}=\beta_{2}=.6$, which are identical to the values in the large-effect conditions used by Kenny et al. (2003) and Bauer et al. (2006).

\section{Data generation}

We mimicked the example of students cross-classified by schools and neighborhoods for data generation. The initial predictor $X$ associated with neighborhoods was drawn from a standard normal distribution. The mediator $M$ associated with schools was generated on the basis of the MM model, as shown in Eq. 1. The parameter values were chosen to be close to the estimates from the empirical demonstration $\left(\sigma_{\mu}^{2}=.2\right.$, and $\sigma_{r}^{2}=$ .7). The outcome $Y$ associated with students was generated using the CC model, as shown in Eq. $2\left(\sigma_{\delta}^{2}=.1\right.$, $\sigma_{\tau}^{2}=.1$, and $\sigma_{e}^{2}=.8$ ). 
Table 6 Standardized bias and coverage rates

\begin{tabular}{|c|c|c|c|c|c|c|c|}
\hline \multirow[t]{2}{*}{ Sample Size } & \multirow[t]{2}{*}{ Degree of CC } & \multirow[t]{2}{*}{ Indirect Effect } & \multirow[t]{2}{*}{ Mean of Estimates } & \multirow[t]{2}{*}{$S D$ of Estimates } & \multirow[t]{2}{*}{ Standardized Bias } & \multicolumn{2}{|c|}{ Coverage Rate } \\
\hline & & & & & & Analytic & Monte Carlo \\
\hline \multirow[t]{4}{*}{200} & \multirow[t]{2}{*}{ Full } & .05 & .046 & .117 & -.034 & $99.9 \%$ & $99.4 \%$ \\
\hline & & .36 & .365 & .442 & .011 & $95.8 \%$ & $94.5 \%$ \\
\hline & \multirow[t]{2}{*}{ Partial } & .05 & .051 & .076 & .013 & $97.6 \%$ & $95.2 \%$ \\
\hline & & .36 & .358 & .163 & -.012 & $92.7 \%$ & $93.1 \%$ \\
\hline \multirow[t]{4}{*}{500} & \multirow[t]{2}{*}{ Full } & .05 & .044 & .057 & -.105 & $99.4 \%$ & $97.0 \%$ \\
\hline & & .36 & .363 & .249 & .012 & $94.4 \%$ & $93.5 \%$ \\
\hline & \multirow[t]{2}{*}{ Partial } & .05 & .049 & .042 & -.024 & $95.0 \%$ & $94.5 \%$ \\
\hline & & .36 & .359 & .102 & -.002 & $92.9 \%$ & $93.1 \%$ \\
\hline \multirow[t]{4}{*}{2,000} & \multirow[t]{2}{*}{ Full } & .05 & .044 & .043 & -.139 & $94.8 \%$ & $95.0 \%$ \\
\hline & & .36 & .348 & .233 & .052 & $95.0 \%$ & $94.6 \%$ \\
\hline & \multirow[t]{2}{*}{ Partial } & .05 & .048 & .027 & -.074 & $93.7 \%$ & $93.6 \%$ \\
\hline & & .36 & .359 & .064 & -.016 & $96.2 \%$ & $96.2 \%$ \\
\hline
\end{tabular}

$\mathrm{CC}$, cross-classification

In summary, the simulation design employed a $3 \times 2 \times 2$ factorial design. In all, 1,000 data sets were generated under each condition. On the basis of the generated data, the indirect effect was computed using the proposed method, and the confidence intervals were calculated using both the analytical and the Monte Carlo approaches.

\section{Analysis}

To examine the bias in the estimated indirect effect, we computed the standardized bias by

$B(\hat{\theta})=\frac{\overline{\hat{\theta}}-\theta}{S(\hat{\theta})}$,

where $\overline{\hat{\theta}}$ is the mean of the indirect effect estimate, $\theta$ the true indirect effect value, and $S(\hat{\theta})$ the standard deviation of the estimates based on the 1,000 replications. The standardized bias is analogous to Cohen's $d$, because it measures the standardized distance between the estimates and the parameters. We consider standardized biases less than .14 to be negligible, because when Cohen's $d$ is less than .14, more than $90 \%$ of the area covered by the two sampling distributions is overlapped (or less than $10 \%$ is not overlapped; Cohen, 1988, pp. 22-23).

To examine the accuracy of the statistical inferences, we computed the coverage rate of the $95 \%$ confidence intervals. The coverage rate of the confidence intervals is the proportion of the obtained confidence intervals that includes the true parameter value. The coverage rate of a $95 \%$ confidence interval should be approximately equal to .95 , with a margin of error of $.0135 .^{1}$ In other words, between $93.65 \%$ and $96.35 \%$ of the obtained confidence intervals should contain the true parameter value. A coverage rate that is higher than $96.35 \%$ indicates decreased power or an inflated Type II error rate, as more replications will fail to find significant results. A coverage rate that is lower than $93.65 \%$ indicates an inflated Type I error rate, as more replications will incorrectly find significant results.

\section{Results}

The results in Table 6 show that the mean standardized biases under all conditions were small, indicating that the indirect effects can be estimated consistently with little bias. The coverage rates of the $95 \%$ confidence intervals were acceptable under all the conditions except for the conditions in which small sample size (e.g., 200) was combined with a high degree of cross-classification and a small effect size. Under such a condition, the coverage rates of both the analytical and Monte Carlo confidence intervals were above $99 \%$, indicating that the Type II error rate was inflated. When the sample size increased to 500 , the coverage rate of the analytical confidence interval was still unacceptably high (99.4\%), but the Monte Carlo confidence interval dropped to $97 \%$, which was only slightly above the upper boundary of the acceptable level (i.e., $96.35 \%$ ).

\footnotetext{
${ }^{1}$ Using the formula provided by Burton, Altman, Royston, and Holder (2006), margin of error $=1.96 \sqrt{p}(1-p) / B$, where $p$ is the nominal coverage rate $(p=.95)$ and $B$ is the number of replications $(B=1,000)$.
} 


\section{Discussions and conclusions}

Individuals live in multiple social contexts, and the factors in one context may influence factors in other contexts and subsequently impact human development. Therefore, it is important to consider social contexts in combination rather than separately, as in traditional neighborhood or school or worksite research (Cook, 2003). However, a big challenge to empirical researchers is that the data structure is often complex when considering multiple contexts simultaneously. In this study, we reviewed existing methods for three common mediation designs in cross-classified data (i.e., $1 \rightarrow 1 \rightarrow 1$, $2^{(\mathrm{A})} \rightarrow 1 \rightarrow 1$, and $2^{(\mathrm{A})} \rightarrow 2^{(\mathrm{A})} \rightarrow 1$ ) and proposed a new method for estimating indirect effects with the more challenging $2^{(\mathrm{A})} \rightarrow 2^{(\mathrm{B})} \rightarrow 1$ design. The proposed method uses both MM models and CC models to analyze mediation effects.

Through a simulation study, we showed that the proposed method can provide a consistent estimate of the true population indirect effect and that its statistical inferences were reliable with a reasonably adequate sample size (i.e., 500 Level-1 units and 50 clusters in each of the crossed factors). The method may not work well when the sample size is small (i.e., 200 Level-1 units and 20 clusters) and the degree of classification is high. This is because the $2^{(\mathrm{A})} \rightarrow 2^{(\mathrm{B})}$ path cannot be assessed in the completely balanced condition. As the degree of crossclassification increases, the data become more and more balanced, causing the estimation of the $2^{(\mathrm{A})} \rightarrow 2^{(\mathrm{B})}$ path to become more and more unstable. In addition, researchers need to have caution when using analytic confidence intervals, because they may have less power than Monte Carlo confidence intervals when the sample size is small.

When applying the proposed method, researchers should be thoughtful about how to choose the weights in the MM model. In this study, we constructed the weights of neighborhoods on the basis of the proportions of students in a school from each neighborhood. This approach is based on the assumption that a neighborhood's impact is proportionate to its size. This assumption may not always be tenable. For example, some small but highly influential neighborhoods may change a school's climate dramatically. In this case, a different weighting system should be considered to better represent the differential impacts of clusters in one factor on a cluster in the other factor.

\section{Limitations and future directions}

The ultimate goal of mediation analysis is to uncover causal pathways through which the cause produces effects. The proposed approach follows the parametric tradition in mediation analysis, in which the path coefficients are estimated on the basis of parametric models (Baron \& Kenny, 1986). As was pointed out by Pearl (2014), this approach has two major drawbacks when it is used to estimate causal mediation.
First, the validity of such estimation methods depends on the assumption of no unmeasured confounders. Although the confounders of the $X-Y$ and $X-M$ relationships can be removed by randomized treatment assignment, such randomization does not remove confounders of the $M-Y$ relationship. Second, this method assumes that the effect of the mediator on the outcome is constant, regardless of the level at which we hold the predictor (i.e., no interaction).

To address the limitations of this approach for estimating causal mediation, we propose three directions for future research. First, this approach could be combined with other methods that are designed for making causal inferences in observational studies, such as propensity score methods. Second, the present model could be extended to incorporate an interaction effect between the predictor and the mediator. Third, mediation with cross-classified multilevel data should be investigated in the framework of nonparametric counterfactual analysis (Imai, Keele, \& Yamamoto, 2010; Pearl, 2014).

\section{Appendix A: Mplus syntax}

1. The $1 \rightarrow 1 \rightarrow 1$ design

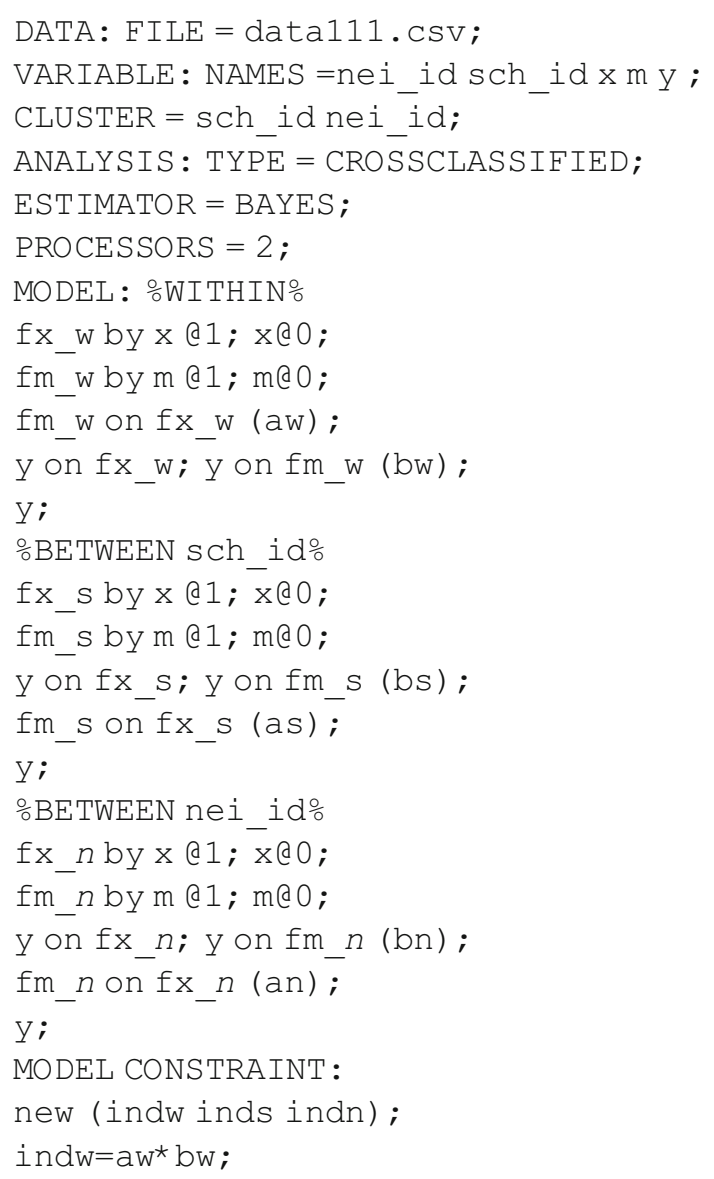


inds $=\mathrm{as} \mathrm{s}^{*} \mathrm{bs}$;

indn=an* bn;

2. The $2^{(\mathrm{A})} \rightarrow 1 \rightarrow 1$ design

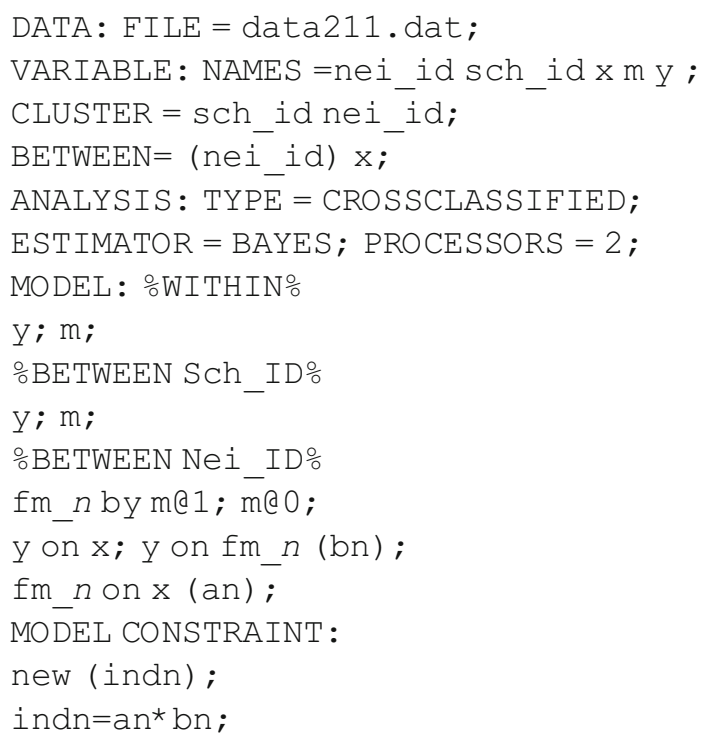

3. The $2^{(\mathrm{A})} \rightarrow 2^{(\mathrm{A})} \rightarrow 1$ design

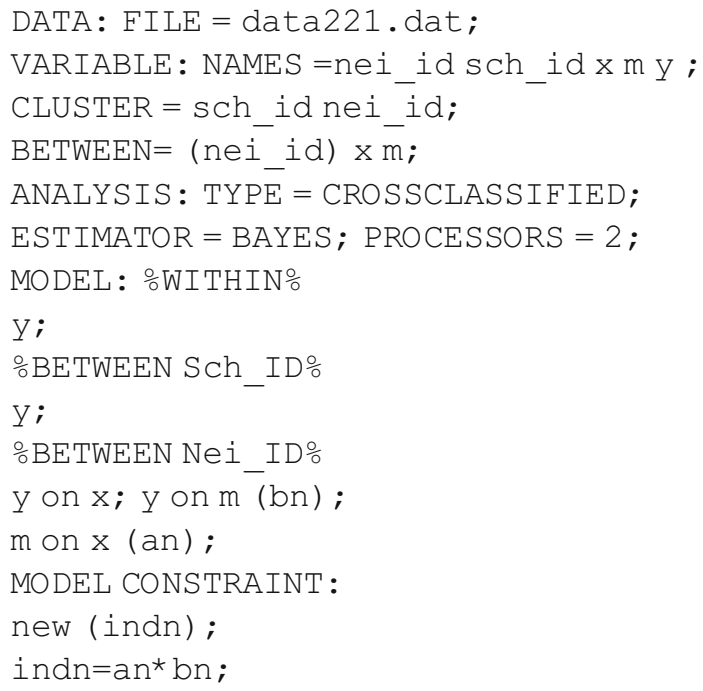

\section{Appendix B: SAS syntax}

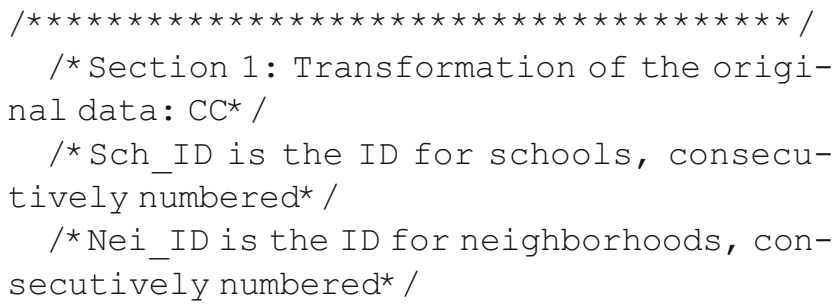

/*X is the initial predictor associated with neighborhood* /

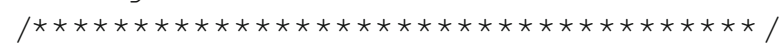


else freq=freq;

if weight $=$. then weight $=0$;

else weight=weight;

drop total freq;

run;

proc sql;

create table temp as

select *

from sch nei_all, nei

where sch_nei__all.Nei_ID=nei.Nei_ID;

quit;

I* 128 is the total number of neighborhoods* /

data sch_nei_wide;

array weì [128] w1-w128;

array Xscore[ 128] X1-X128;

mean_ $\mathrm{X}=0$;

do $i=1$ to 128 until (last.Sch_ID);

set temp;

by Sch_ID;

wei[ i] =weight;

$\mathrm{Xscore}[\mathrm{i}]=\mathrm{X}$;

mean_X=mean_X+wei[ i] *Xscore[ i] ;

end;

run;

data sch_nei_wide;

set sch nei wide;

drop i Nei_id weight XX1-X128;

run;

proc sql;

create table MM as

select *

from sch_nei_wide, sch

where sch_nei_wide.Sch_ID =sch.Sch_ID;

\section{quit;}

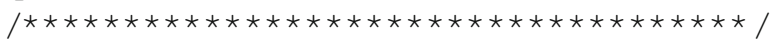

/* Section 2: Model Estimation* /

/*Estimate the $2(\mathrm{~A})->2$ (B) path using

Multiple Membership model* /

/* w1-w128 are the weight variables* /

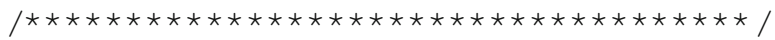

proc mixed data $=\mathrm{MM}$ asycov covtest ;

model $M=$ mean_X/solution ddfm=kr covb;

random $\mathrm{w} 1-\mathrm{w} 1 \overline{2} 8 /$ type=toep $(1)$;

ods output solutionF=fix_mm;

run;

/*Estimate the 2 (B) $->1$ path using CCREM* /

proc mixed data $=\mathrm{CC}$ asycov covtest ;

class Sch_ID Nei_ID;

model $\mathrm{y}=\bar{M} \mathrm{X} / \mathrm{solution} \mathrm{ddfm}=\mathrm{kr}$ covb;

random intercept / subject $=S c h \_I D ;$

random intercept / subject $=\mathrm{Nei} I D$;

ods output solutionF=fix_CC; run;

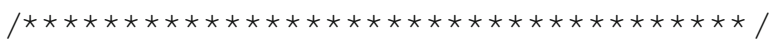

/* Section 3: Compute indirect effect and confidence intervals* /

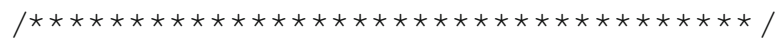

PROC IML;

/*Collecting necessary model estimates

from fixed effects and G matrix* /

USE FIX_mm;

READ ALL VAR \{ EFFECT\} INTO PARAMSmm;

READ ALL VAR \{ ESTIMATE $\}$ INTO ESTmm;

READ ALL VAR $\{$ STDERR $\}$ INTO SEmm;

$\mathrm{aMM}=\mathrm{ESTmm}[$ 2] ;

$\mathrm{SEaMM}=\mathrm{SEmm}[$ 2] ;

USE FIX_CC ;

READ ALL VAR $\{$ EFFECT $\}$ INTO PARAMSCC;

READ ALL VAR \{ ESTIMATE $\}$ INTO ESTCC;

READ ALL VAR \{ STDERR $\}$ INTO SECC;

$\mathrm{bCC}=\operatorname{ESTCC}[2]$;

$\mathrm{SEbCC}=\mathrm{SECC}[2]$;

/* Computing analytical CI* /

$\mathrm{Eab}=\mathrm{aMM} \mathrm{MCC}^{\star}$;

$\mathrm{Vab}=(\mathrm{aMM} \star \star 2) *(\mathrm{SEbCC} \star * 2)+$

$(\mathrm{b} \mathrm{C} \mathrm{C} \star \star 2) \star(\mathrm{SE} \mathrm{a} \mathrm{M} \mathrm{M} \star \star 2)+$

$\left(\mathrm{SEaMM}^{\star} \star 2\right) *(\mathrm{SEbCC} \star * 2)$;

Ind_LCL $=E a b-1.96 * S Q R T(V a b) ; * 95 \%$

Confidence limits;

Ind_UCL=Eab + 1.96* SQRT (Vab) ;

analytic=Eab | | ind_LCL | ind_UCL;

C R E A T a n a l y t i C F R M analytic[ COLNAME $=\{$ Indirect Ind_LCL_ANA Ind_UCL_ANA\} ] ;

APPEND FROM analytic;

/* Compute Monte Carlo CI* /

Estimates=aMM || $\mathrm{bCC}$;

zero $=\{0\}$;

$\mathrm{A}=\mathrm{SEaMM} \mid$ | zero;

$\mathrm{B}=$ zero $\mid$ SEbCC ;

$\mathrm{L}=\mathrm{A} / \mathrm{B}$;

$\mathrm{Z}=\operatorname{rannor}(\mathrm{J}(\mathbf{1 0 0 0}, \mathbf{2}, \mathbf{0})) ;$ * starts with standard normal;

Sim_Est $=Z^{\star} L+J(1000,1,1) *$ Estimates;

Sim_Ind=Sim_Est $[, 1]$ \#Sim_Est $[$, 2] ;

$\mathrm{CREATE} \quad \mathrm{S} i \mathrm{~m}$ - i $\mathrm{nd} \quad \mathrm{FROM}$

Sim_ind[ COLNAME $=\{$ Indirect $\}]$;

APPEND FROM Sim_ind;

QUIT;

proc sort data=Sim_ind; by Ind; run;

data rank; set Sim ind;

if $N_{-}=(.05 / 2) * 1000$ then output;

if $N_{-}=(1-.05 / 2) \star 1000$ then output;

run; 
proctranspose data $=r a n k$

out=ind_centilesprefix=Ind_MC_CL;

var Indirect; run;

data Indirect_result;

merge analytic ind_centiles ;

drop name_;

run;

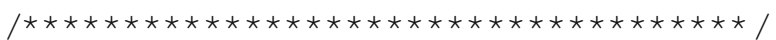

/* Section 4: Print out results*/

/*Fix_MM has estimates from the Multiple Membership model* /

/* Fix CC has estimates from the CCREM* /

/*Indirect_result has five columns* /

/*Col 1: Indirect effect; $\operatorname{Col} 2-3$ :

Analytical CI; Col 4-5: Monte Carlo CI* /

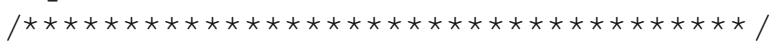

proc print data $=$ Fix mm;

proc print data $=\mathrm{Fix} \_\mathrm{CC}$;

proc print data=indirect_result;

run;

\section{References}

Ainsworth, J. W. (2002). Why does it take a village? The mediation of neighborhood effects on educational achievement. Social Forces, $81,117-152$.

Asparouhov, T., \& Muthen, B. (2006, August). Multilevel modeling of complex survey data. Paper presented at the Joint Statistical Meeting in Seattle, ASA Section on Survey Research Methods, Seattle, WA.

Asparouhov, T., \& Muthén, B. (2012, July). General random effect latent variable modeling: Random subjects, items, contexts, and parameters. Paper presented at the annual meeting of the National Council on Measurement in Education, Vancouver, British Columbia, Canada.

Baron, R. M., \& Kenny, D. A. (1986). The moderator-mediator variable distinction in social psychological research: Conceptual, strategic, and statistical considerations. Journal of Personality and Social Psychology, 51, 1173-1182. doi:10.1037/0022-3514.51.6.1173

Bauer, D. J., Preacher, K. J., \& Gil, K. M. (2006). Conceptualizing and testing random indirect effects and moderated mediation in multilevel models: New procedures and recommendations. Psychological Methods, 11, 142-163. doi:10.1037/1082-989X.11.2.142

Brault, M., Janosz, M., \& Archambault, I. (2014). Effects of school composition and school climate on teacher expectations of students: A multilevel analysis. Teaching and Teacher Education, 44, 148-159. doi:10.1016/j.tate.2014.08.008

Browne, W., \& Goldstein, H. (2001). Multiple membership multiple classification (MMMC) models. Statistical Modelling, 1, 103-124.

Burton, A., Altman, D. G., Royston, P., \& Holder, R. L. (2006). The design of simulation studies in medical statistics. Statistics in Medicine, 25, 4279-4292. doi:10.1002/sim.2673

Chandola, T., Clarke, P., Wiggins, R. D., \& Bartley, M. (2005). Who you live with and where you live: Setting the context for health using multiple membership multilevel models. Journal of Epidemiology and Community Health, 59, 170-175.

Cohen, J. (1988). Statistical power analysis for the behavioral sciences (2nd ed.). Hillsdale, NJ: Erlbaum.

Cook, T. D. (2003). The case for studying multiple contexts simultaneously. Addiction, 98, 151-155.

Imai, K., Keele, L., \& Yamamoto, T. (2010). Identification, inference and sensitivity analysis for causal mediation effects. Statistical Science, 25, 51-71.

Kenny, D. A., Korchmaros, J. D., \& Bolger, N. (2003). Lower level mediation in multilevel models. Psychological Methods, 8, 115128. doi:10.1037/1082-989X.8.2.115

Krull, J. L., \& MacKinnon, D. P. (2001). Multilevel modeling of individual and group level mediated effects. Multivariate Behavioral Research, 36, 249-277.

Luo, W., Cappaert, K. J., \& Ning, L. (2015). Modelling partially crossclassified multilevel data. British Journal of Mathematical and Statistical Psychology, 68, 342-362. doi:10.1111/bmsp.12050

Maas, C. J. M., \& Hox, J. J. (2005). Sufficient sample sizes for multilevel modeling. Methodology, 1, 86-92. doi:10.1027/1614-2241.1.3.86

MacKinnon, D. P., Lockwood, C. M., \& Williams, J. (2004). Confidence limits for the indirect effect: Distribution of the product and resampling methods. Multivariate Behavioral Research, 39, 99-128. doi: 10.1207/s15327906mbr3901_4

Muthén, L. K., \& Muthén, B. O. (1998-2012). Mplus user's guide (7th ed.). Los Angeles, CA: Muthén \& Muthén.

Pearl, J. (2014). Interpretation and identification of causal mediation. Psychological Methods, 19, 459-481. doi:10.1037/a0036434

Preacher, K. J., Zyphur, M. J., \& Zhang, Z. (2010). A general multilevel SEM framework for assessing multilevel mediation. Psychological Methods, 15, 209-233. doi:10.1037/a0020141

Rasbash, J., \& Goldstein, H. (1994). Efficient analysis of mixed hierarchical and crossed random structures using a multilevel model. Journal of Educational and Behavioral Statistics, 19, 337-350.

Selig, J. P., \& Preacher, K. J. (2008, June). Monte Carlo Method for Assessing Mediation: An interactive tool for creating confidence intervals for indirect effects [Computer software]. Available from http://quantpsy.org/

Zhang, Z., Zyphur, M. J., \& Preacher, K. J. (2009). Testing multilevel mediation using hierarchical linear models: Problems and solutions. Organizational Research Methods, 12, 695-719. doi:10.1177/ 1094428108327450 